\title{
Observable majoron emission in neutrinoless double beta decay
}

\author{
Z.G. Berezhiani ${ }^{1,2}$ \\ Sektion Physik, Universität München, W-8000 Munich 2, FRG \\ and Institute of Physics of the Georgian Academy of Sciences, 380077 Tbilisi, Georgia
}

\author{
A.Yu. Smirnov ${ }^{3}$ \\ Institute for Nuclear Research, Russian Academy of Sciences, 117312 Moscow, Russian Federation
}

and

\author{
J.W.F. Valle ${ }^{4,5}$ \\ Instituto de Fisica Corpuscular - CSIC, Departament de Fisica Tè̀rica, Universitat de València, \\ E-46100 Burjassot, Valencia, Spain
}

Received 8 July 1992

\begin{abstract}
We consider a class of simplest majoron models where neutrino-majoron couplings can be in the range $g \sim 10^{-5}-10^{-3}$, leading to the observability of neutrinoless double beta decay with majoron emission. The majoron is a singlet (or mostly a singlet) of the electroweak $S U(2) \otimes U(1)$ gauge symmetry, thus avoiding conflict with the LEP data on Z-decay, which rules out the triplet and doublet majoron models. Some aspects of the phenomenology of such models are discussed.
\end{abstract}

\section{Introduction}

An attractive approach to the neutrino mass problem is to consider neutrinos as Majorana particles with small masses arising as a result of spontaneous lepton violation [1]. In these models a massless Goldstone boson, the majoron, should exist. Several realizations of this idea were proposed. They are distinguished mainly by the weak isospin $(I)$ properties of the majoron and have different phenomenology: the singlet majoron with $I=0$ [2], the triplet one with $I=1$ [3] and the doublet majoron $I=\frac{1}{2}$ [4].

If the majoron Yukawa couplings with neutrinos are sufficiently strong $\left(g \sim 10^{-5}-10^{-3}\right)$, this would imply several interesting consequences for particle

\footnotetext{
1 Alexander von Humboldt Fellow.

2 Internet address:ZURAB@hep.physik.uni-muenchen.de.

3 Internet address:SMIRNOV@inucres.msk.su.

4 Bitnet address: VALLE@EVALUN11.

5 Decnet address:16444::VALLE.
}

physics and astrophysics. Perhaps the most striking is the possibility of substantial neutrinoless double beta decay rates with majoron emission, denoted $\left(2 \beta_{0 v J}\right)$ [5]. On the other hand, the majoron can play a significant role for the history of the early universe, in the evolution of stellar objects, and in supernovae astrophysics. Neutrino decay with majoron emission may also be relevant in connection with the solar neutrino problem [6-9]. Finally, the existence of neutrino masses in excess of $50 \mathrm{eV}$ or so, such as the $17 \mathrm{keV}$ neutrino [10-12], if confirmed, can be considered as a strong argument in favour of the existence of such a majoron, since only fast decays (or annihilation) of such neutrinos into majorons can avoid conflict with cosmological and astrophysical restrictions $[1,13]$.

The existence of majorons with nontrivial weak isospin properties, such as the triplet and doublet majorons would lead to appreciable neutrino-majoron $(\nu-J)$ couplings. However these are strongly excluded by recent LEP data [14] on the invisible width 
of the $Z$-boson. Indeed, they would contribute to this width through the decay $Z \rightarrow \rho+J$, where $\rho$ is a scalar partner of the majoron $J$, the equivalent of 2 and $\frac{1}{2}$ extra neutrino species, respectively, whereas the LEP limit on the number of active light neutrino species allows only the three known neutrinos, to a few percent accuracy. On the other hand the singlet "seesaw" majoron [2] is extremely weakly coupled with neutrinos. This would suggest that it is meaningless to search for the effects of a large $\nu-J$ coupling, such as the search for $2 \beta_{0 \nu J}$ decay.

In this letter we show that there is not direct contradiction between the absence majoron emission in $Z$-boson decay and the possibility of fast $2 \beta_{0 \nu J}$ decay. The former is determined by a gauge coupling and the weak isospin content of the majoron, whereas the latter is related only to the majoron Yukawa coupling. We present a variety of simple models in which the majoron, being a singlet or predominantly singlet of the electroweak $S U(2) \otimes U(1)$ symmetry, nevertheless can be sufficiently strongly coupled with neutrinos. The LEP data on Z-decay are automatically reconciled with the presence of a strong enough $\nu_{e^{-}}$ majoron coupling, in the range $g_{e e} \sim 10^{-5}-10^{-3}$, which leads to experimentally observable $2 \beta_{0 \nu J}$ decay rates. Moreover, this could have consequences for neutrino fluxes from gravitational collapse [15].

The paper is organized as follows. In section 2 the status of neutrinoless double beta decays is reviewed in the context of the majoron models and the general idea on the possibility of strong $\nu-J$ coupling is discussed. In section 3 different models of singlet (or dominantly singlet) majoron are presented as a realizations of this possibility. In section 4 we draw our conclusions.

\section{General considerations}

In order to have strong neutrino-majoron $\nu_{e}-J$ couplings, the scale of spontaneous lepton number violation has to be sufficiently low. This generates the majoron, denoted $J$, and originated from some neutral complex scalar singlet $\sigma$, defined through

$\sigma=\frac{1}{\sqrt{2}}(v+\rho+\mathrm{i} J)$.

Below this scale $v$ the relevant terms in the lagrangian are the neutrino mass term and the Yukawa couplings with the majoron $J$ and its scalar partner $\rho$ :

$L_{\text {mass }}=-\frac{1}{2} m \nu_{\mathcal{c}}^{\mathrm{T}} C \nu_{e}+$ h.c. ,

$L_{\mathrm{Yuk}}=\frac{1}{2} \mathrm{i} g J \nu_{e}^{\mathrm{T}} C \nu_{e}+\frac{1}{2} g \rho \nu_{e}^{\mathrm{T}} C \nu_{e}+$ h.c.,

where $C$ is the matrix of charge conjugation and the majoron coupling $g$ is given as

$g=m / v$

independent of the specifics of the majoron model ${ }^{\# 1}$.

Recent data on neutrinoless double beta decay yield [16]

$m \lesssim 1-3 \mathrm{eV}, \quad g \lesssim 10^{-4}-10^{-3}$,

where the bounds vary depending on the evaluation of nuclear matrix elements. The lower and upper values in $\left(3^{\prime}\right),\left(3^{\prime \prime}\right)$ correspond to the models of ref. [17] and ref. [18] respectively. The sensitivity expected in planned experimental searches should improve these limits by an order of magnitude. Then eq. (3) implies that a positive result in $2 \beta_{0 \nu J}$ decay searches is expected only if $v \lesssim 10-100 \mathrm{keV}$. This implies that in neutrinoless double beta decay not only the massless majoron $J$ is emitted but also the accompanying $0^{+}$scalar partner $\rho$ of mass $m_{\rho} \lesssim v$. As a result we expect the existence of new features in the electron sum energy spectrum, as indicated in fig. 1 . Indeed, close to the endpoint $Q$, i.e., between $Q-m_{\rho}$ and $Q$, only the massless majoron is emitted, while in the region from 0 to $Q-m_{\rho}$ both $\rho$ and $J$ are emitted. In principle this would imply a deficit of events close to the endpoint.

It is interesting to note that for $v$ in this range the $2 \beta_{0 \nu J}$ decay should be faster as compared to the $2 \beta_{0 \nu}$ one. Indeed, taking into account that the nuclear matrix elements for both processes are actually the same one has

$\frac{\Gamma\left(2 \beta_{0 \nu J}\right)}{\Gamma\left(2 \beta_{0 v}\right)} \simeq \frac{1}{84 \pi^{2}}\left(\frac{Q}{v}\right)^{2} R(x)$,

where $R(x)$ is the corresponding phase space factor [5]. Since the typical mass difference $Q$ between the initial and final nuclei is a few $\mathrm{MeV}$, it follows that

\#1 In the case of three neutrino flavours $m$ is the $m_{e e}$ element of the neutrino Majorana mass matrix, and $g=g_{e e}$ is a corresponding coupling constant. 


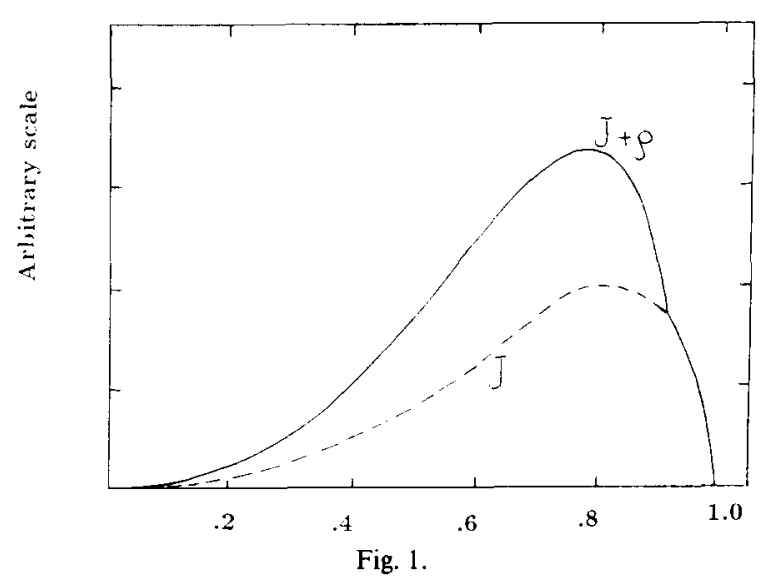

the above ratio exceeds one for $v \leqslant 10-100 \mathrm{keV}$.

The simplest scheme containing a singlet majoron includes one isosinglet lepton which acquires a large mass from its interaction with $\sigma$, after spontaneous lepton number violation [2]. If $v \gtrsim Q \sim 1 \mathrm{MeV}$ then the $2 \beta_{0 \nu J}$ decay is strongly suppressed due to the smallness of $g \sim m_{\nu} / v \lesssim 10^{-5}-10^{-6}$. On the other hand if the scale $v \ll Q$ the contributions of the mass eigenstate neutrinos cancel so that both matrix elements $g_{c e}$ and $m_{e c}$ are zero and the probabilities of both types of neutrinoless $2 \beta$ decays, $2 \beta_{0 \nu J}$ and $2 \beta_{0 \nu}$ vanish. In addition, this is in conflict with the cosmological nucleosynthesis constraints. Indeed, due to the strong $\nu J$ interactions the right handed neutrinos together with the majoron $J$ and its scalar partner $\rho$ should come to equilibrium in the early universe before $t=1$ $s$ and thereby significantly affect the stage of primordial nucleosynthesis. On the other hand the effective number of light neutrino species allowed by the primordial ${ }^{4} \mathrm{He}$ abundance is $N_{\nu}<3.4$ [19], in agreement with just the three conventional neutrinos. In contrast, in view of the astrophysical upper limit on the majoron coupling to electrons that follows from stellar energy loss arguments [20], the triplet and doublet majorons were considered as promising candidates for $2 \beta_{0 \nu /}$ decay. However, these models are now ruled out by LEP data. As a result we will consider alternative schemes with and without isosinglet neutrinos that can lead to substantial majoron emission rates in neutrinoless double beta decay (section $3)$.

The lowest dimension effective operator, relevant

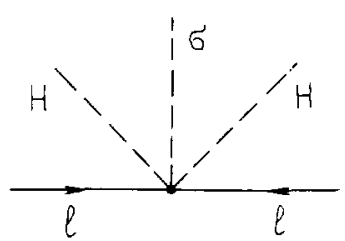

Fig. 2.

for the generation of Majorana neutrino masses, is the folling (see fig. 2 ) $\# 2$.

$\mathscr{L}=G_{J} l{ }^{\mathrm{T}} C \tau_{\alpha} l H^{\mathrm{T}} \tau_{\alpha} H \sigma+$ h.c.,

where $\sigma$ is an $\mathrm{SU}(2) \otimes \mathrm{U}(1)$ singlet with $L=-2, l=$ $\left(\begin{array}{c}\nu_{e} \\ e\end{array}\right)_{L}$ is a lepton doublet $H=\left(\begin{array}{c}H^{+}+ \\ H^{\circ}\end{array}\right)$ is the standard Higgs doublet, and $\tau_{\alpha}, \alpha=1,2,3$ are the isospin Pauli matrices. After $\mathrm{SU}(2) \otimes \mathrm{U}(1)$ breaking by $\left\langle H^{0}\right\rangle=$ $\left(2 \sqrt{2} G_{\mathrm{F}}\right)^{-1 / 2} \simeq 188 \mathrm{GeV}$, eq. (5) implies that $g=G_{J}\langle H\rangle^{2}$ is the effective coupling constant $g$.

As we emphasized above, the existence of strong $\nu_{e}-J$ coupling requires a sufficiently low scale of lepton number violation $\langle\sigma\rangle \ll\langle H\rangle$. In this connection a question appears concerning the stability of this hierarchy. First note that this is not the usual gauge hierarchy, as far as $\sigma$ is a gauge singlet. Its couplings with the Higgs field $H$ in the Higgs potential cannot be generated with gauge boson loops. On the other hand the radiative corrections to the mass term of $\sigma$ that follow from eq. (5) are of the order of $\delta M_{\sigma}^{2} \sim$ $\left(\mathrm{g}^{2} / 32 \pi^{2}\right) \Lambda^{2}$, where $A$ is some physical cut off. Taking into account the experimental bounds on the neutrino mass and requiring that $v \sim \delta M_{\sigma}$ this corresponds to $g \sim 10^{-3}-10^{-5}$ for $A$ in the range $20-200$ $\mathrm{GeV}$. In particular models, considered in section 3, there may be other contributions to $\delta M_{\sigma}^{2}$, that should be taken into account. Although we will not consider in great detail these radiative corrections, we would like stress that, from general arguments, in these singlet majoron models the possibility to have $\langle\sigma\rangle \ll\langle H\rangle$ does not necessarily require fine tuning, unlike the case of the triplet and doublet majoron models ${ }^{\# 3}$. Although this question deserves special consideration [21], here we wish to note that a cutoff in the required range may be naturally provided.

\#2 Generalization to the case of three lepton doublets is straightforward.

\#3 For the latter a fine tuning, at least for the splitting of the neutral component from the charged ones, was unavoidable. 
We now consider mechanisms where the effective operator eq. (5) arises as the low energy limit of new renormalizable interactions, in analogy with the way in which the Fermi four fermion interaction emerges from $W$ and $Z$ boson exchange.

\section{Models}

The effective operator in eq. (5) may arise at the tree level from the exchange of a neutral heavy lepton (fig. $3 \mathrm{a}$ ). Alternatively, it can appear as a radiative correction due to the effects of new Higgs bosons as in fig. $3 \mathrm{~b}$ and fig. $3 \mathrm{c}$. It may also arise from tree level heavy Higgs triplet exchange (fig. 3d). Let us discuss now these possibilities.

\subsection{Singlet majoron in the " $\mu$-model"}

This model [22] is a variant of the "seesaw" model with the heavy Dirac lepton suggested in ref. [23]. Here the operator eq. (5) can be effectively induced through the "exchange" of heavy Dirac neutral fermion carrying one unit of lepton number (see fig. 3a). The relevant terms in the lagrangian are the bare Dirac mass term and the Yukawa couplings ${ }^{\# 4}$

$$
h_{\nu} l^{\mathrm{T}} C \nu^{\mathrm{c}} H+M \nu^{\mathrm{c}} C S+f \sigma S^{\mathrm{T}} C S+\text { h.c. }
$$

\#4 A possible $\nu^{\mathrm{c}} \nu^{\mathrm{c}} \sigma^{*}$ entry would not give a mass to the light neutrinos and can be forbidden by demanding supersymmetry.
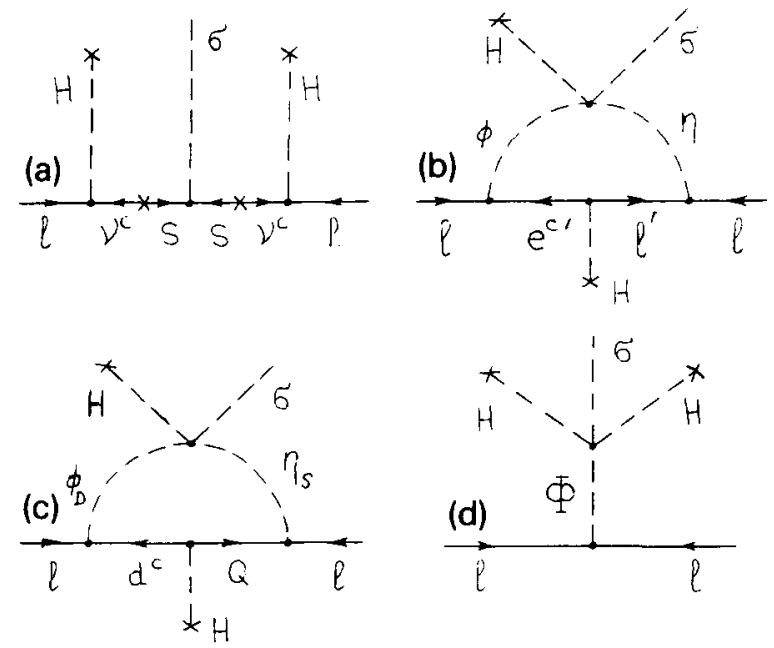

Fig. 3.
The first coupling generates the Dirac mass term $D=h_{\nu}\langle H\rangle$, while the second term gives the Majorana mass term $\mu$ for the isosinglet $S$ that violates lepton number by two units, $\mu=f\langle\sigma\rangle$. The full mass matrix in Majorana basis $\nu, \nu^{c}, S$ can be written as $\# 5$

$\left(\begin{array}{ccc}0 & D & 0 \\ D & 0 & M \\ 0 & M & \mu\end{array}\right)$.

For small values of the parameter $\mu \ll D \ll M$, the heavy leptons here are of quasi-Dirac type and the Majorana mass of the light, mostly isodoublet neutrino $\nu_{\mathrm{L}}$ arising from eq. (7) and the $\nu_{e}$-majoron coupling constant are, respectively

$m=\mu\left(\frac{D}{M}\right)^{2}, \quad g=\frac{m}{v}=f\left(\frac{d}{M}\right)^{2}$.

Note the different relationship between $m_{\nu}$ and $\mu \lesssim\langle\sigma\rangle$, the lepton number breaking scale. This is a crucial feature of this model, which contrasts with the simplest seesaw model. It follows from the fact that the model contains a quasi-Dirac heavy lepton whose mass $\sim M$, is invariant under lepton number and unrelated to neutrino masses. In contrast, the minimal seesaw model has a heavy Majorana lepton whose mass is inversely related to that of the isodoublet neutrino. In both cases the heavy lepton admixture in the weak charged current is determined by the ratio $D / M$. However, in the " $\mu$-model" this value is restricted only by weak universality constraints, and not by limits on neutrino masses [1] \#6. As a result a $\nu_{e^{-}}$ $J$ coupling as large as $10^{-3}$ can be achieved for values of $D / M \lesssim 0.1$, well consistent with present weak universality constraints [1].

The phenomenology of this model was considered in detail in several papers [1]. For masses below $m_{Z}$ the heavy quasi-Dirac leptons may be singly produced at LEP 1, giving rise to striking events characterized by a large amount of missing energy $[24,25]$. The existence of such neutral heavy leptons at higher

\#5 For the generalization to three neutrino families one introduces six isosinglet neutrinos, three $\nu^{\mathrm{c}}$-type and three $S$-type.

\#6 The crucial point is that the heavy lepton mass here arises mostly from the entry $M$, invariant under lepton number, unlike the case of the seesaw model. As a result $M$ can be relatively low without implying too large $m_{\nu}$ values. In fact, in the limit where the lepton number is exact neutrino masses are strictly forbidden [23]. 
masses can at present only be probed due to their indirect effects. For example, if we include mixing between the various generations we have the interesting possibility of flavour and/or $C P$ violation [26-28] even in the limit where $\mu \rightarrow 0$ and the isodoublet neutrinos become strictly massless. As a result, processes such as $\mu \rightarrow e \gamma, \tau \rightarrow e \gamma, \mu-e$ conversion in nuclei, $Z \rightarrow e \tau$, etc. are all allowed. Moreover, the corresponding rates are restricted only by the precision of weak universality tests. As a result, they all can be within the sensitivity of present as well as those expected at the upcoming tau factory [29].

\subsection{Singlet majoron in the Zee model}

This model is a variant to the original Zee model [30] which introduces the spontaneous violation of lepton number [13] and, as a consequence, the existence of a massless pseudoscalar majoron. The relevant terms in the lagrangian are the Yukawa couplings $\$ 7$

$\frac{m_{i}}{\langle H\rangle} \Gamma_{i} H e_{\mathrm{R} i}+h_{i j} \Gamma_{i} \phi e_{\mathrm{R} i}+f_{i j} l_{i}^{\mathrm{T}} \mathrm{Ci} \tau_{2} l_{j} \eta^{+}+$h.c.,

where $i, j=e, \mu, \tau$. The first term is the canonical one responsible for generating the charged lepton masses $m_{i}$ when the $\mathrm{SU}(2) \otimes \mathrm{U}(1)_{Y}$ gauge symmetry is broken by $\langle H\rangle$. The additional couplings involve another Higgs doublet $\phi$ as well as the Zee singlet $\eta$ and are specified by matrices $f, h$ (in generation space), $f$ being antisymmetric. In addition we use the following quartic term in the scalar potential:

$\lambda H \tau_{2} \phi \eta^{+} \sigma+$ h.c.

instead of the usual cubic term $A H \tau_{2} \phi \eta^{+}$that would explicitly violate lepton number. The quartic coupling fixes $L(\sigma)=2=-L(\eta)$ and induces a mixing between the physical singly charged scalars which plays a crucial role in the radiative generation of neutrino mass and of the $\nu_{e}-J$ coupling. Indeed, the effective operator in eq. (5) arises at one loop level, see fig. $3 \mathrm{~b}$, and has the following associated coupling constant

$g=G_{J}\langle H\rangle^{2}=\frac{h_{e i} f_{i e} \lambda}{16 \pi^{2}} \frac{\langle H\rangle m_{i}}{M_{\phi}^{2}-M_{\eta}^{2}} \ln \frac{M_{\phi}^{2}}{M_{\eta}^{2}}$,

\#7 For simplicity we assume that all the Yukawa coupling constants are real. where $M_{\phi}^{2}$ and $M_{\eta}^{2}$ are the eigenvalues of the mass matrix of charged scalars $\phi^{-}$and $\eta^{+}$in the limit of small mixing. From fig. $3 \mathrm{~b}$ it is clear that if we require natural flavour conservation in neutral Higgs couplings ( $h_{i j}$ diagonal) then $g=0$. Therefore we now relax this requirement and allow nondiagonal flavour changing couplings for $\phi$ in eq. (11).

In order to estimate the maximal possible value of the induced $\nu_{e}-J$ coupling constant in this model we first note that the dominant contribution will come from $\tau$ lepton exchange in the loop. Next, we must discuss the relevant constraints. First note that the exchange of the scalar $\eta^{+}$gives a contribution to the decay width of the $\tau$-lepton, $\tau \rightarrow e \bar{\nu}_{e} \nu_{\tau}$ given as

$\Gamma_{\tau}=\Gamma_{\tau}^{\mathrm{SM}}\left(1+2 \rho_{\tau}\right), \quad \rho_{\tau}=\frac{f^{2} / M^{2}}{8 \sqrt{2} G_{\mathrm{F}}}$.

The present experimental limit $\rho_{t} \lesssim 2.5 \%$ then implies $f / M_{\eta}^{+} \lesssim 0.33 /\langle H\rangle$. Similarly, $\phi^{+}$exchange leads to a new contribution to the process $e^{+} e^{-} \rightarrow$ $\nu \tau \bar{\nu}_{\tau}$. While this process is invisible, the related process $e^{+} e^{-} \rightarrow \nu_{\tau} \bar{\nu}_{\tau} \gamma$ can be seen. The limit from the ASP experiment [32] then implies $h / M_{\phi^{+}} \lesssim 2.2 /\langle H\rangle$. One can now easily estimate that the maximum attainable values for $g$ (which occurs for $M_{\phi} \simeq M_{\eta}$ ) consistent with the restrictions above and with the recent LEP limits on charged scalar masses is

$g=G_{J}\langle H\rangle^{2} \lesssim \frac{f h \lambda}{16 \pi^{2}} \frac{m_{\tau}}{M_{\eta}} \frac{\langle H\rangle}{M_{\phi}} \lesssim 4 \times 10^{-5} \lambda$.

So, in this model, provided that $\lambda<1$, a sufficiently large value for the $\nu_{e^{-}} J$ coupling constant within the sensitivity of neutrinoless double beta decay experiments can only be obtained by adjusting all the parameters at their allowed borders.

\subsection{Singlet majoron in a coloured Zee model}

This is a variant of the previous model which can easily allow for larger $\nu_{e}-J$ coupling in $2 \beta_{0 \nu J}$ decay. The relevant terms in the lagrangian are

$h_{i j} l_{i}^{\mathrm{T}} C b_{L, j}^{\mathrm{c}} \phi_{\mathrm{D}}+f_{i j} l_{i}^{\mathrm{T}} C Q_{L j} \eta_{\mathrm{S}}+\lambda H \tau_{2} \phi_{\mathrm{D}} \eta_{\mathrm{s}} \sigma+$ h.c.,

where $i, j=1,2,3$ and $\phi_{\mathrm{D}}=\left(\begin{array}{c}\phi^{2 / 3}-1 / 3 \\ \phi\end{array}\right)$ and $\eta_{\mathrm{S}}$ are colour triplet leptoquark scalars: $\phi_{\mathrm{D}}$ is an $\mathrm{SU}(2) \otimes \mathrm{U}(1)$ doublet with $Y=\frac{1}{3}$ and $L=-1$ while $\eta_{\mathrm{S}}$ is a singlet 
with $Y=\frac{2}{3}$ and also $L=-1$. Again lepton number is spontaneously broken by $\langle\sigma\rangle$ and this generates the majoron, as in the previous case. The key contribution to the effective operator in eq. (5) now arises (see fig. $3 \mathrm{c}$ ) from $b$-quark exchange in the loop. One obtains

$g=G_{J}\langle H\rangle^{2} \approx \frac{3 h_{e b} f_{b e} \lambda}{16 \pi^{2}} \frac{m_{b}\langle H\rangle}{M_{\phi_{\mathrm{D}}}^{2}-M_{\eta \mathrm{S}}^{2}} \ln \frac{M_{\phi_{\mathrm{D}}}^{2}}{M_{\eta \mathrm{S}}^{2}}$

in a similar notation as a above. This contribution is enhanced with respect to the one in eq. (11) by a factor $\sim 3 m_{b} / m_{\tau}, 3$ coming from colour. In addition, flavour changing neutral current mediated interactions are automatically forbidden at the tree level since there is only one isodoublet Higgs contributing to generating the masses of the charged fermions. The constraints on the allowed $\nu_{e}-J$ coupling strength now arise from the contribution of $\eta^{+1 / 3}$ exchange in $b$ meson semileptonic decays. Considering that the uncertainties in the limits on $b$-meson decays are somewhat higher than in the $\tau$ lepton case one can easily obtain the maximum attainable values for the $\nu_{c}-J$ coupling consistent with experiment as

$g=G_{J}\langle H\rangle^{2} \lesssim 10^{-3} \lambda$.

Thus, for reasonable choices of parameters, we can have in this model a large enough value for the $\nu_{e}-J$ coupling constant as to be of interest in neutrinoless double beta decay experiments.

\subsection{Singlet-triplet majoron}

For completeness we note that it is also possible to generate the desired $\nu-J$ interaction in a scheme where the majoron is not a pure $\mathrm{SU}(2) \otimes U(1)$ singlet. The most straightforward example is to invoke a tripletsinglet scheme, suggested in ref. [33]. The model contains the following couplings:

$f l^{\mathrm{T}} C \tau_{\alpha} \Phi_{\alpha} l+\lambda \Phi_{\alpha}^{*} H \tau_{\alpha} H \sigma+$ h.c. ,

where $\Phi_{a}=\left(\Phi^{++}, \Phi^{+}, \Phi^{0}\right), a=1,2,3$ is a weak isospin triplet carrying hypercharge $Y=2$ and lepton number $L=-2$. In this case the $\nu-J$ coupling arises from $\Phi$ exchange, as shown in fig. $3 \mathrm{~d}$. In order to avoid an excessive contribution to the invisible $Z$-boson width, the neutral component $\Phi^{0}$ should be heavier than about $40 \mathrm{GeV}$, whereas the lower limits on the charged components should be somewhat stronger.

In such a model the majoron is given as [33]

$J \simeq \frac{1}{v}\left(\langle\sigma\rangle \operatorname{Im} \sigma+\langle\Phi\rangle \operatorname{Im} \Phi^{0}\right)$,

where $v=\sqrt{\langle\sigma\rangle^{2}+\langle\Phi\rangle^{2}}$ is the scale of lepton number violation. In order to avoid an excessive contribution to the invisible $Z$ width we need to require $\langle\Phi\rangle\langle\langle\sigma\rangle\langle 10-100 \mathrm{keV}$. The first inequality may be achieved if the VEV of the triplet arises only from the quartic interaction term present in eq. (16). Such an induced VEV is given as $\langle\Phi\rangle \sim \lambda v\langle H\rangle^{2} / M_{\Phi}^{2}$. The second inequality is needed in order to have a sizeable $\nu_{c} \nu_{c} J$ coupling. With this it follows that the electron-majoron coupling is automatically too small to give any significant contribution to stellar energy loss. Although phenomenologically viable, we do not favour this model because of the fine-tunings necessary in order to have a sizeable majoron coupling, a situation reminiscent of the old triplet majoron model.

\section{Conclusions}

LEP data on Z-decays do not exclude the possibility of observable rates for majoron emission in neutrinoless double beta decays. The majoron coupling to the $Z$ boson is determined by a gauge coupling and weak isospin, whereas its coupling to neutrinos responsible for majoron double beta decays is of Yukawa origin. In general these couplings are not related. We have presented models where the LEP restrictions are avoided because the majoron is a singlet, or dominantly singlet under the electroweak $\mathrm{SU}(2) \otimes \mathrm{U}(1)$ gauge symmetry. At the same time the effective coupling $g$ of the singlet majoron to the $\nu_{e}$ can be as large as $g \sim 10^{-3}-10^{-5}$. The key points of these models with large neutrino-majoron couplings are the existence of new heavy leptons and/or scalar bosons at the electroweak scale with the spontaneous violation of lepton number occurring below 10-100 $\mathrm{keV}$. In the most appealing model (section 3.1) the effective neutrino-majoron coupling arises from the exchange of a neutral quasi-Dirac heavy lepton (fig. $3 a$ ). We also discussed the possibility of inducing this coupling from radiative corrections associated with 
additional Higgs bosons (figs. $3 b$ and $3 c$ ) or tree-level Higgs exchange (fig. 3d). Of these models where the enhanced majoron-neutrino coupling arises mostly from scalar boson exchange the best possibility is the coloured Zee model.

\section{Acknowledgement}

We thank A.S. Barabash, G. Fiorentini, M.I. Vysotsky and M.G. Shchepkin for discussions.

\section{References}

[1] J.W.F. Valle, Prog. Part. Nucl. Phys. 26 (1991) 91 and references therein.

[2] Y. Chikashige, R. Mohapatra and R. Peccei, Phys. Lett. B 98 (1981) 265.

[3] G. Gelmini and M. Roncadelli, Phys. Lett. B 99 (1981) 411.

[4] A. Santamaria and J.W.F. Valle, Phys. Lett. B 195 (1987) 423; Phys. Rev. Lett. 60 (1988) 397; Phys. Rev. D 39 (1989) 1780;

C. Aulakh and R. Mohapatra, Phys. Lett. B 119 (1982) 136.

[5] H. Georgi, S. Glashow and S. Nussinov, Nucl. Phys. B 193 (1981) 297

[6] J. Frieman, H. Haber and K. Freese, Phys. Lett. B 200 (1988) 115.

[7] J. Bahcall, S. Petcov, S. Toshev and J.W.F. Valle, Phys. Lett. B 181 (1986) 369.

[8] Z. Berezhiani and M. Vysotsky, Phys. Lett. B 199 (1988) 281.

[9]Z. Berezhiani et al., Ferrara preprint INFN-FE-05/91 (1991), Z. Phys. C, to appear [JETP Lett. 55 (1992) 151].

[10] A. Hime and J. Simpson, Phys. Rev. D 39 (1989) 1825; D. 39 (1989) 1837.

[11] A. Hime and H. Jelley, Phys. Lett. B 257 (1991) 441.

[12] F. Boehm, in: Electroweak physics beyond the standard model, ed. J.W.F. Valle and J. Velasco (World Scientific, Singapore, 1992).
[13] G. Gelmini, S. Nussinov and R. Peccei, UCLA preprint UCLA-91-TEP-15 (1991).

[14] J. Steinberger, in: Electroweak physics beyond the standard model, eds. J.W.F. Valle and J. Velasco (World Scientific, Singapore, 1992).

[15] Z. Berezhiani and A. Smirnov, Phys. Lett. B 220 (1989) 279;

K. Choi and A. Santamaria, Phys. Rev. D 42 (1990) 293.

[16] H.V. Klapdor, J. Phys. G Suppl. 17 (1991) S129; M. Doi et al., Phys. Rev. D 35 (1988) 2575; A.S. Barabash, Phys. Lett. B 216 (1989) 257; A. Vasenko et al., Mod. Phys. Lett. A 5 (1990) 1299.

[17] K. Grotz and H.V. Klapdor, Nucl. Phys. A 460 (1986) 395.

[18] J. Engel, P. Vogel and M. Zirnbauer, Phys. Rev. C 37 (1988) 731.

[19] K. Olive et al., Phys. Lett. B 236 (1990) 454.

[20] J.E. Kim, Phys. Rev. 150 (1987) 1.

[21 ] Z. Berezhiani, A. Smirnov and J.W.F. Valle, in preparation.

[22] M.C. Gonzalez-Garcia and J.W.F. Valle, Phys. Lett. B 216 (1989) 360.

[23] R. Mohapatra and J.W.F. Valle, Phys. Rev. D 34 (1986) 1642.

[24] M. Dittmar, M.C. Gonzalez-Garcia, A. Santamaria and J.W.F. Valle, Nucl. Phys. B 332 (1990) 1.

[25] M.C. Gonzalez-Garcia, A. Santamaria and J.W.F. Valle, Nucl. Phys. B 342 (1990) 108.

[26] J. Bernabeu, A. Santamaria, J. Vidal, A. Mendez and J.W.F. Valle, Phys. Lett. B 187 (1987) 303.

[27] G.C. Branco, M.N. Rebelo and J.W.F. Valle, Phys. Lett. B 225 (1989) 385.

[28] N. Rius and J.W.F. Valle, Phys. Lett. B 246 (1990) 249.

[29] M.C. Gonzalez-Garcia and J.W.F. Valle, Mod. Phys. Lett. A 7 (1992) 477.

[30] A. Zee, Phys. Lett. B 93 (1980) 389.

[31] R. Barbieri and L. Hall, Nucl. Phys. B 364 (1991) 27; Z. Berezhiani, unpublished;

G. Leontaris, C. Vayonakis and J. Vergados, preprint IOA269 (1992).

[32] C. Hearty et al., Phys. Rev. Lett. 58 (1987) 1711.

[33] J. Schechter and J.W.F. Valle, Phys. Rev. D 25 (1982) 774. 